\title{
The expression of Duffy antigen receptor for chemokines by epithelial ovarian cancer decreases growth potential
}

\author{
QINYI ZHU, LU JIANG and XIPENG WANG \\ Department of Gynecology, Shanghai First Maternity and Infant Hospital, \\ Tongji University School of Medicine, Shanghai 201204, P.R. China
}

Received November 18, 2015; Accepted March 3, 2017

DOI: $10.3892 / \mathrm{ol} .2017 .5954$

\begin{abstract}
Epithelial ovarian cancer (EOC) is one of the main causes of cancer-associated mortality in females with gynecological malignancies. Duffy antigen receptor for chemokines (DARC) has previously been reported to be involved in tumor growth and the inhibition of tumor metastasis. However, the association between DARC and EOC remains unknown. The aim of the present study was to investigate the expression of DARC in the SKOV3 human epithelial ovarian cancer cell line with the establishment of a subcutaneous model in nude mice. To investigate the effects of DARC on the tumorigenesis of human epithelial ovarian cancer cells, GV287-DARC-L.V lentiviral vectors containing a DARC overexpression construct were transfected into SKOV3 cells. The present study revealed that transfection with DARC reduced the viability of SKOV3 cells in vitro by performing an MTT assay. SKOV3-DARC and SKOV3-negative control (NC) cells cultured in vitro were injected into nude mice to establish a subcutaneous model. The ovarian tumor volumes and the tumor weights were observed. Immunohistochemistry to detect CD31 expression was used to determine the microvessel density (MVD) in SKOV3-DARC and SKOV3-NC tumors. The results of the present study revealed that DARC-induced inhibition of tumor growth was associated with MVD in xenograft tumors. This suggested that DARC was a negative regulator of tumor growth in EOC, primarily via the inhibition of tumor angiogenesis.
\end{abstract}

\section{Introduction}

Epithelial ovarian cancer (EOC) affects females worldwide, and is the fifth highest cause of mortality from malignancy in

Correspondence to: Professor Xipeng Wang, Department of Gynecology, Shanghai First Maternity and Infant Hospital, Tongji University School of Medicine, 2699 West Gaoke Road, Shanghai 201204, P.R. China

E-mail: Dr_xwang@hotmail.com

Key words: Duffy antigen receptor for chemokines, ovarian cancer, animal model, nude mice, microvessel density females (1). The majority of patients with EOC are diagnosed at an advanced clinical stage, since they are asymptomatic until the tumor has metastasized on the surface of the peritoneum (2). Surgery combined with chemotherapy is the main strategy for treatment, but this has a poor outcome. Furthermore, $75 \%$ of patients with advanced stage EOC develop recurrences and succumb to mortality within 5 years from the point of diagnosis $(3,4)$. The histological subtypes of EOC include mucinous, serous, endometrioid and clear cell carcinoma (5). The pathogenesis of EOC is a complex process that involves interactions among chemokines, chemokine receptors, environmental influences, inflammatory cells, hereditary factors and cancer suppression and progression mechanisms (6).

Chemokines and chemokine receptors have previously been studied due to their involvement in cancer progression, including proliferation, migration, invasion and angiogenesis (7,8). C-X-C motif chemokine ligand 8 (CXCL8), C-C motif chemokine ligand (CCL) 2 and CCL5 have previously been reported to be associated with cancer progression $(9,10)$. Chemokines may be mediated by transcriptional activity and posttranslational regulation (10). Duffy antigen receptor for chemokines (DARC) is an atypical chemokine binder, incapable of transmitting signals via the classic G-protein-mediated signaling pathways. DARC is able to efficiently internalize its cognate chemokine ligands and act as a scavenger. Multiple previous studies have demonstrated that DARC serves an inhibitory function in breast cancer growth and progression by sequestration of pro-malignancy chemokines (11-13). However, the association between DARC and EOC remains unknown.

Chemokines promote angiogenesis by interaction with EOC cells. In EOC, tumor derived vascular endothelial growth factor promotes tumor angiogenesis (14). Angiogenesis is essential for tumor growth (15). DARC is widely expressed on vascular endothelial cells and erythrocytes (16). DARC is also present on blood vessels and serves as a systemic barrier to tumor metastasis (16). Given the broad distribution of DARC within the body and its inhibitory effect on tumor progression and metastasis, the present study hypothesized that DARC may inhibit ovarian cancer growth and progression by angiogenesis. In order to investigate this hypothesis, the present study established a nude mouse model with subcutaneous primary ovarian cancer. The biological mechanism of DARC was subsequently analyzed. 


\section{Materials and methods}

Cell culture. The SKOV3 human ovarian cancer cell line was obtained from the American Type Culture Collection (Manassas, VA, USA). The SKOV3 cells were cultured in Dulbecco's modified Eagle's medium (DMEM; HyClone; GE Healthcare Lifesciences, Logan, UT, USA) supplemented with 5\% penicillin-streptomycin (Gibco; Thermo Fisher Scientific, Inc., Waltham, MA, USA) and 10\% fetal bovine serum (FBS; Gibco; Thermo Fisher Scientific, Inc.). The SKOV3 cells were transfected with the lentiviral vector GV287-DARC-L.V-GFP (LVKL7847-2; Genechem Co., Ltd., Shanghai, China) containing a DARC overexpression construct, with the sequence: 5' GAGGATCCCCGGGTACCGGTCGCCACC ATGGGGAACTGTCTGCACAGG 3', and an empty vector negative control (NC) GV287-NC-L.V-GFP (KL3120-1; Genechem Co., Ltd.) in the presence of $5 \mu \mathrm{g} / \mathrm{ml}$ polybrene (Sigma-Aldrich; Merck KGaA, Darmstadt, Germany) and ENi.S (Genechem Co., Ltd.) for $16 \mathrm{~h}$, following which the medium was replaced by DMEM supplemented with $10 \%$ FBS. The infection efficiency of lentivirus was determined under a Zeiss LSM 510 laser scanning confocal fluorescence microscope (Zeiss GmbH, Jena, Germany) $72 \mathrm{~h}$ following infection (Fig. 1). The cells were counted manually in 5 fields of view. The DARC-transfected SKOV3 cells and mock-transfected SKOV3 cells were incubated at $37^{\circ} \mathrm{C}$ with $5 \% \mathrm{CO}_{2}$.

MTT assay. For the MTT assay, the SKOV3-DARC and SKOV3-NC cells were seeded at a density of 2,000 cells/well in 96-well plates $72 \mathrm{~h}$ following transfection. Subsequently, MTT reagent ( $20 \mu \mathrm{l}, 5 \mathrm{mg} / \mathrm{ml}$, cat. no., ab211091; Abcam, Cambridge, MA, USA) was added to each well and incubated for $1 \mathrm{~h}$ at $37^{\circ} \mathrm{C}$. The remaining crystals were dissolved in dimethyl sulfoxide. The absorbance was evaluated at $492 \mathrm{~nm}$. The assays were performed in triplicate.

Murine model of ovarian cancer. A total of 10 female athymic nude mice (4-6 weeks old) weighing 15-18 g were purchased from Shanghai JiaoTong University School of Medicine (Shanghai, China) and housed at $18-20^{\circ} \mathrm{C}$ and a constant humidity of $60-70 \%$, with ad libitum feeding schedules and as 12/12 $\mathrm{h}$ light/dark cycle. The nude mice were bred under specific pathogen free conditions. Protocols for the treatment of nude mice were approved by the committee of Shanghai First Maternity and Infant Hospital, Tongji University School of Medicine (Shanghai, China). The nude mice were randomly separated into two groups as follows: The DRAC group (DARC-transfected SKOV3 cells) and NC group (mock-transfected SKOV3 cells). To establish a subcutaneous model, nude mice from each group were injected with $0.10 \mathrm{ml}$ DMEM containing $1 \times 10^{6}$ SKOV3-DARC or SKOV3-NC cells. Mice were observed every week for tumor growth and appearance, and tumors were harvested 4 weeks following injection. Tumor diameter and tumor weights were recorded. The volume $(\mathrm{V})$ of ovarian tumors was evaluated by the following formula: $\mathrm{V}=1 / 2 \mathrm{x}$ length $\mathrm{x}$ width ${ }^{2}$. Tumor tissues were fixed in paraformaldehyde for frozen slide preparation.

Immunohistochemistry. For immunohistochemical analysis of the tumor sections, tumor samples were collected 4 weeks following injection and processed for frozen section analysis. Sections ( $8 \mu \mathrm{m}$ thick) were prepared as follows: Tumor tissues isolated from nude mice were fixed in $4 \%$ paraformaldehyde, sectioned and mounted on slides. The slides were fixed in cold acetone for $15 \mathrm{~min}$ without antigen retrieval. Following washing with PBS, endogenous peroxide was blocked with 3\% $\mathrm{H}_{2} \mathrm{O}_{2}$ in PBS for 15 min at $20-22^{\circ} \mathrm{C}$. Slides were blocked with $5 \%$ bovine serum albumin (Sigma-Aldrich; Merck KGaA) and $10 \%$ normal goat serum (Sigma-Aldrich; Merck KGaA) in PBS for $50 \mathrm{~min}$ at room temperature, followed by incubation with primary antibody (mouse monoclonal CD31; cat. no., Ab28364; dilution, 1:80; Abcam, Cambridge, MA, USA) in PBS overnight at $20^{\circ} \mathrm{C}$. Secondary antibody (Biotin-SP-conjugated Affinipure goat anti-rabbit immunoglobulin G; cat. no., A8275; dilution, 1:350; Sigma-Aldrich; Merck KGaA ) in PBS was added for $45 \mathrm{~min}$ at $37^{\circ} \mathrm{C}$ and horseradish peroxidase (HRP; dilution, 1:500; Sigma-Aldrich; Merck KGaA) was added for $35 \mathrm{~min}$ at $37^{\circ} \mathrm{C}$. Subsequently, HRP was detected using 3,3'-diaminobenzidine (DAB) as a substrate with a $100 \mathrm{sec}$ incubation period at $20-22^{\circ} \mathrm{C}$, followed by washing and counter-staining with hematoxylin (Sigma-Aldrich; Merck KGaA). Microvessel density (MVD) was determined from five random high power fields using light microscopy. Vessels with a well-defined lumen or linear vessel shape were observed for blood microvessel analysis. The negative control was prepared by replacing CD31 with PBS under the same conditions as the primary antibody.

Statistical analysis. Statistical analyses were performed using SPSS version 22.0 software (IBM SPSS, Armonk, NY, USA). Data were expressed as the mean \pm standard deviation. An unpaired Student's t-test was used to determine P-values. Levene's test was used to assess the homogeneity of variance. Continuous variables in the figures were presented as the mean \pm standard error of the mean. $\mathrm{P}<0.05$ was considered to indicate a statistically significant difference.

\section{Results}

Overexpression of the DARC molecule in SKOV3 cell line. To investigate the anti-malignant functional significance of DARC in ovarian cancer, a DARC-overexpressing SKOV3 cell line was generated in vitro (Fig. 1, at x200 magnification). The infection efficiency of SKOV3 cells was evaluated by observing concomitant green fluorescent protein (GFP) expression. There was no significant difference between GFP expression of SKOV3-DARC and SKOV3-NC cells observed $(\mathrm{P}>0.05)$.

DARC inhibited EOC viability in vitro. To determine whether DARC expression affected the viability of SKOV3 cells in vitro, an MTT assay was performed. DARC overexpression demonstrated a significant effect on cell viability when compared with SKOV3-NC cells. The number of SKOV3-DARC cells was significantly decreased $\left(1.19 \times 10^{4} \pm 21.01\right)$ compared with SKOV3-NC cells $\left(1.38 \times 10^{4} \pm 366.09 ; \mathrm{P}=0.001\right.$; Fig. 2$)$.

DARC inhibited EOC development in vivo. To investigate the inhibitory function of DARC on tumor development, the present study established a subcutaneous model to observe 


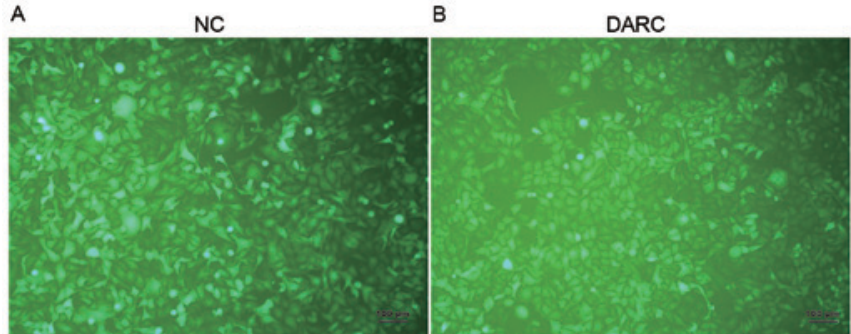

Figure 1. Expression of SKOV3-DARC and SKOV3-NC cells by stable transfection. (A) The infection efficiency of NC-transfected SKOV3 cells was determined using a fluorescent microscope. (B) The infection efficiency of DARC-transfected SKOV3 cells was evaluated using a fluorescent microscope. Scale bar, $100 \mu \mathrm{m}$. DARC, Duffy antigen receptor for chemokines; $\mathrm{NC}$, negative control.

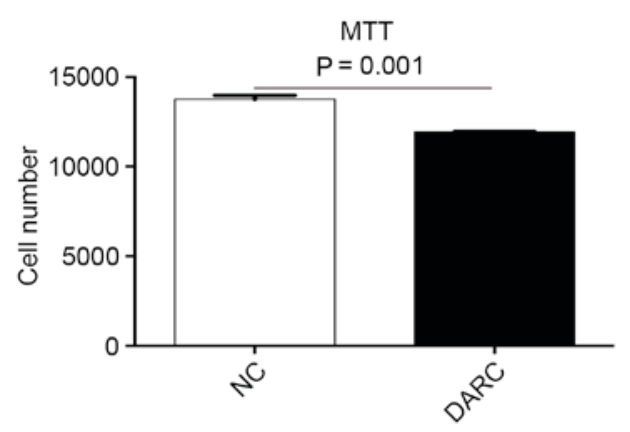

Figure 2. DARC inhibited epithelial ovarian cancer cell viability in vitro. The number of SKOV3-DARC cells was significantly lower compared with SKOV3-NC cells. DARC, Duffy antigen receptor for chemokines; NC, negative control.

ovarian cancer growth in nude mice $(\mathrm{n}=5)$. Nude mice were injected with $0.10 \mathrm{ml}$ DMEM containing $1 \times 10^{6} \mathrm{SKOV} 3-\mathrm{DARC}$ or SKOV3-NC cells. The experimental group demonstrated decreased tumor volume $\left(\mathrm{mm}^{3}\right)$ on day 21 and 28 post-injection (Fig. 3). The tumor volume of the SKOV3-DARC group $\left(21.24 \pm 4.75 \mathrm{~mm}^{3}\right)$ was significantly lower at day 21 compared with the SKOV3-NC group $\left(67.48 \pm 36.13 \mathrm{~mm}^{3} ; \mathrm{P}=0.045\right)$. At day 28, the tumor volume of the SKOV3-DARC group $\left(36.30 \pm 16.38 \mathrm{~mm}^{3}\right)$ was also lower compared with that of the SKOV3-NC group $\left(158.43 \pm 108.66 \mathrm{~mm}^{3} ; \mathrm{P}=0.038\right)$. Furthermore, the present study compared the tumor weight between the SKOV3-DARC and SKOV3-NC groups at the time of sacrifice (Fig. 4). The tumor weight of the SKOV3-DARC group $(0.55 \pm 0.13 \mathrm{~g})$ was lower compared with the SKOV3-NC group $(1.40 \pm 0.72 \mathrm{~g})$ on day $28(\mathrm{P}=0.032 ; \mathrm{Fig}$. 4$)$. The tumors of the SKOV3-DARC group were, in general, smaller compared with those of the SKOV3-NC group (Fig. 5). Therefore, it was revealed that DARC expression regulated ovarian cancer cell proliferation in vivo.

DARC inhibited EOC progression by anti-angiogenesis in vivo. Tumor growth and progression depend on angiogenesis. To determine whether DARC expression-induced inhibition of cancer growth was associated with tumor angiogenesis, CD31 staining in tumor sections was performed. MVD was analyzed at X200 magnification. The expression of CD31 was confined to the cytomembrane of vascular endothelial cells (Fig. 6). MVD in the DARC group $(11.04 \pm 1.76)$ was lower compared

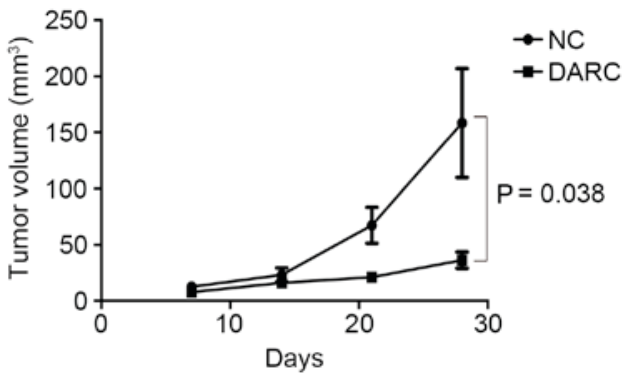

Figure 3. Tumor volume of the DARC group and NC group in the murine subcutaneous tumor model. The tumor volume $\left(\mathrm{mm}^{3}\right)$ of the DARC group was significant lower compared with the NC group. (Volume=1/2xlengthxwidth ${ }^{2}$ ). DARC, Duffy antigen receptor for chemokines; NC, negative control.

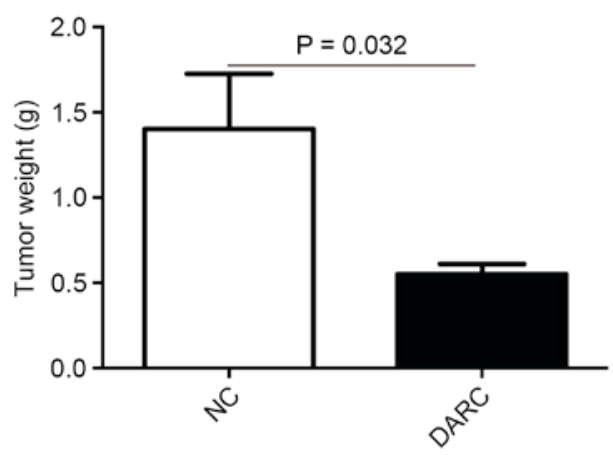

Figure 4. Tumor weight of the DARC group and NC group in the mice subcutaneous model. The tumor weight $(\mathrm{g})$ of the DARC group mice was significantly lower compared with the NC group mice. DARC, Duffy antigen receptor for chemokines; NC, negative control.

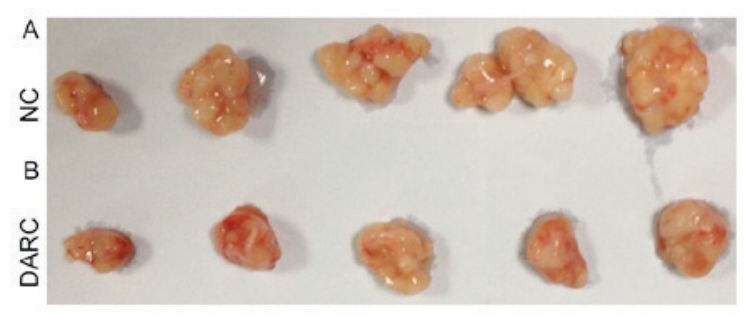

Figure 5. Image of the DARC group and NC group tumors. The tumors of the (A) NC group were larger compared with those of the (B) DARC group. DARC, Duffy antigen receptor for chemokines; NC, negative control.

with the NC group $(17.32 \pm 3.55)$ in tumor sections of nude mice $(\mathrm{P}=0.008$; Fig. 6). These results revealed that DARC expression inhibited ovarian cancer growth by decreasing tumor angiogenesis.

\section{Discussion}

Chemokines are multifunctional secreted peptides that are involved in regulating leukocyte migration, malignancy progression and metastasis. Chemokines may facilitate tumorigenesis by providing an inflammation microenvironment for tumor cells. The functions of chemokines include directly influencing tumor growth, transformation and metastasis, and indirect functions affecting tumor-leukocyte interactions and tumor angiogenesis. Solid tumors contain various types of 


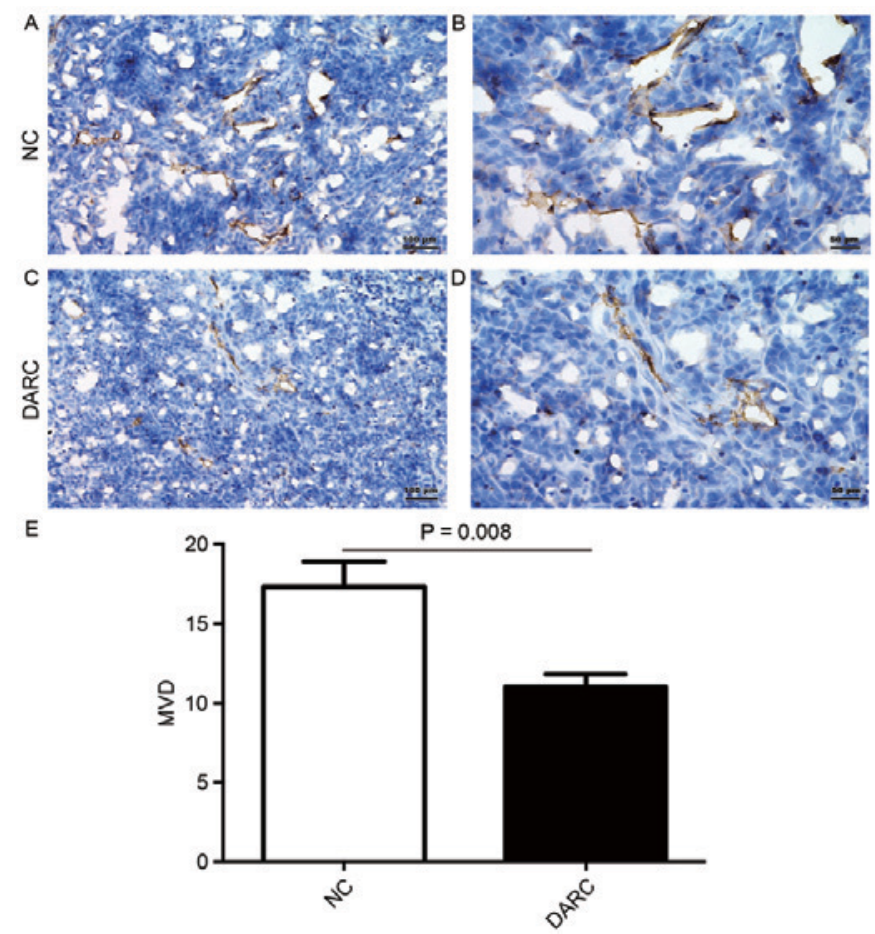

Figure 6. DARC inhibited epithelial ovarian cancer progression by anti-angiogenesis in vivo. (A) The expression of MVD was observed by immunohistochemistry in the NC group at (A) x200 magnification; scale bar, $100 \mu \mathrm{m}$ and (B) at $\mathrm{x} 400$ magnification; scale bar, $50 \mu \mathrm{m}$. (C) The expression of MVD was observed by immunohistochemistry in the DARC group at x 200 magnification; scale bar, $100 \mu \mathrm{m}$ and (D) at $\mathrm{x} 400$ magnification; scale bar, $50 \mu \mathrm{m}$. (E) The MVD of the DARC group was significantly lower compared with the NC group. DARC, Duffy antigen receptor for chemokines; NC, negative control; MVD, microvessel density.

stromal cells, including endothelial cells and fibroblast cells. In addition, tumors are infiltrated with inflammatory cells, including macrophages, neutrophils and lymphocytes. These all contribute to the microenvironment for the growth and metastasis of tumor cells. Chemokines may attract macrophages, which affect tumor progression (17). C-X-C motif chemokine ligand 12 and $\mathrm{C}-\mathrm{X}-\mathrm{C}$ motif chemokine receptor 4 have been demonstrated to be involved in peritoneal metastasis of ovarian cancer and gastric cancer (18). However, the mechanism of these chemokine factors remains unknown.

Atypical chemokine receptors, which include DARC, C-C chemokine receptor type 11 and D6, may act as scavengers and internalize their cognate chemokine ligands. DARC is not only a receptor for the malaria parasites, plasmodium knowlesi and plasmodium vivax, but is also known to be a typical decoy receptor that binds with $\mathrm{CC}$ chemokines and CXC chemokines (19). DARC lacks the Asp-Arg-Tyr motif to enable $\mathrm{G}$ protein coupling in the second intracellular loop $(20,21)$. When a ligand binds to DARC, it doesn't induce $\mathrm{G}$ protein-coupled signal transduction and $\mathrm{Ca}^{2+}$-flux (22). DARC, which is specialized for chemokine sequestration, may regulate chemokine bioavailability by binding its chemoattractant ligands without activating its signal transduction cascades for tumor cell migration $(23,24)$. DARC is unable to induce signaling responses by binding to a ligand so that it appears to be a scavenger of multiple chemokines (25). Thus, it is also proposed to limit tumorigenesis, tumor growth and metastasis.
Certain previous studies have demonstrated that DARC is a negative regulator of lung caner and prostate cancer $(26,27)$. The present study hypothesized that DARC may inhibit the proliferation and growth of human ovarian cancer in vitro and in vivo, which indicated that the intratumor chemokine network may be partially modulated by DARC.

Angiogenic chemokines have been reported to involved in tumor angiogenesis. Certain chemokines may promote an inflammatory microenvironment for tumor initiation, growth, angiogenesis, progression and metastasis, whereas others may suppress tumor proliferation by promoting antitumor immunity (28-30). The capture and clearance of angiogenic chemokines may induce inhibition of tumor growth and proliferation. The present study hypothesized that DARC, as a silent chemokine receptor, is able to selectively bind to angiogenic chemokines and inhibit tumor angiogenesis. MVD, as an indicator of angiogenesis, has been used for the evaluation of tumor angiogenesis in multiple types of human tumors. A previous study revealed that DARC induced tumor neovascularity inhibition in breast cancer (13). The present study demonstrated that the inhibition effect of DARC on tumor progression was associated with MVD.

The present study observed that DARC decreased SKOV3 cell proliferation in vitro. It was also revealed that DARC inhibited SKOV3 cell growth in vivo, and that the SKOV3-DARC tumor volumes were smaller compared with SKOV3-NC tumor volumes. The present study demonstrated that the SKOV3-DARC tumor weights were lower compared with the SKOV3-NC tumor weights. Furthermore, the MVD was confirmed to be positively associated with DARC expression. The present study suggested that DARC may inhibit EOC growth and metastasis via an anti-angiogenesis effect. This result is partially consistent with certain previous studies in other types of cancer, including non-small-cell lung cancer (26), breast cancer $(13,31,32)$, thyroid cancer (20) and laryngeal squamous cell carcinoma (33). The inhibitory effect of DARC on ovarian cancer growth and metastasis is mainly due to its ability to sequester pro-malignant chemokines. Furthermore, the anti-metastatic and anti-angiogenesis functions of DARC were reported to be derived from the interaction between the tumor suppressor gene kangai 1 and DARC on vascular endothelial cells (34).

In conclusion, the present study demonstrated that overexpression of DARC was associated with inhibited tumor growth, proliferation and tumor angiogenesis of ovarian cancer. Further experiments investigating DARC and chemokines are required in the future for exploring their potential as neo-therapeutic targets in EOC.

\section{Acknowledgements}

The present study was supported by the National Science Foundation of China (grant nos. 81372787 and 81072136), the Top 100 Medical Elite in Shanghai (grant no. XBR 2011065) and the Shanghai Municipal Bureau of Health (grant no. 20164Y0024).

\section{References}

1. Siegel R, Naishadham D and Jemal A: Cancer statistics, 2013. CA Cancer J Clin 63: 11-30, 2013. 
2. Weidle UH, Birzele F, Kollmorgen G and Rueger R: Mechanisms and targets involved in dissemination of ovarian cancer. Cancer Genomics Proteomics 13: 407-423, 2016.

3. Lavoué V, Thédrez A, Levêque J, Foucher F, Henno S, Jauffret V, Belaud-Rotureau MA, Catros V and Cabillic F: Immunity of human epithelial ovarian carcinoma: The paradigm of immune suppression in cancer. J Transl Med 11: 147, 2013.

4. Mei L, Chen H, Wei DM, Fang F, Liu GJ, Xie HY, Wang X, Zou J, Han X and Feng D: Maintenance chemotherapy for ovarian cancer. Cochrane Database Syst Rev CD007414, 2013.

5. Tan DS and Kaye S: Ovarian clear cell adenocarcinoma: A continuing enigma. J Clin Pathol 60: 355-360, 2007.

6. Barbolina MV,Kim M, Liu Y, Shepard J, Belmadani A, Miller RJ, Shea LD and Stack MS: Microenvironmental regulation of chemokine (C-X-C-motif) receptor 4 in ovarian carcinoma. Mol Cancer Res 8: 653-664, 2010.

7. Zlotnik A: Chemokines and cancer. Int J Cancer 119: 2026-2029, 2006.

8. Balakin KV, Ivanenkov YA, Tkachenko SE, Kiselyov AS and Ivachtchenko AV: Regulators of chemokine receptor activity as promising anticancer therapeutics. Curr Cancer Drug Targets 8: 299-340, 2008

9. Soria G, Yaal-Hahoshen N, Azenshtein E, Shina S, Leider-Trejo L, Ryvo L, Cohen-Hillel E, Shtabsky A, Ehrlich M, Meshel T, et al Concomitant expression of the chemokines RANTES and MCP-1 in human breast cancer: A basis for tumor-promoting interactions. Cytokine 44: 191-200, 2008.

10. Soria $\mathrm{G}$ and Ben-Baruch A: The inflammatory chemokines CCL2 and CCL5 in breast cancer. Cancer Lett 267: 271-285, 2008.

11. Feng LY, Ou ZL, Wu FY, Shen ZZ and Shao ZM: Involvement of a novel chemokine decoy receptor CCX-CKR in breast cancer growth, metastasis and patient survival. Clin Cancer Res 15: 2962-2970, 2009.

12. Wu FY, Ou ZL, Feng LY, Luo JM, Wang LP, Shen ZZ and Shao ZM: Chemokine decoy receptor d6 plays a negative role in human breast cancer. Mol Cancer Res 6: 1276-1288, 2008.

13. Wang J, Ou ZL, Hou YF, Luo JM, Shen ZZ, Ding J and Shao ZM: Enhanced expression of Duffy antigen receptor for chemokines by breast cancer cells attenuates growth and metastasis potential Oncogene 25: 7201-7211,2006.

14. Zhang L, Yang N, Park JW, Katsaros D, Fracchioli S, Cao G, O'Brien-Jenkins A, Randall TC, Rubin SC and Coukos G: Tumor-derived vascular endothelial growth factor up-regulates angiopoietin-2 in host endothelium and destabilizes host vasculature, supporting angiogenesis in ovarian cancer. Cancer Res 63 3403-3412, 2003.

15. Folkman J: Tumor angiogenesis: Therapeutic implications. N Engl J Med 285: 1182-1186, 1971.

16. Rot A: Contribution of Duffy antigen to chemokine function Cytokine Growth Factor Rev 16: 687-694, 2005

17. Balkwill F: Cancer and the chemokine network. Nat Rev Cancer 4: 540-550, 2004

18. Zhao HB, Tang CL, Hou YL, Xue LR, Li MQ, Du MR and Li DJ: CXCL12/CXCR4 axis triggers the activation of EGF receptor and ERK signaling pathway in CsA-induced proliferation of human trophoblast cells. PLoS One 7: e38375, 2012.

19. Szabo MC, Soo KS, Zlotnik A and Schall TJ: Chemokine class differences in binding to the Duffy antigen-erythrocyte chemokine receptor. J Biol Chem 270: 25348-25351, 1995.
20. Latini FR, Bastos AU, Arnoni CP, Muniz JG, Person RM, Baleotti W Jr, Barreto JA, Castilho L and Cerutti JM: DARC (Duffy) and BCAM (Lutheran) reduced expression in thyroid cancer. Blood Cells Mol Dis 50: 161-165, 2013.

21. Horne K and Woolley IJ: Shedding light on DARC: The role of the Duffy antigen/receptor for chemokines in inflammation, infection and malignancy. Inflamm Res 58: 431-435, 2009.

22. Neote K, Mak JY, Kolakowski LF Jr and Schall TJ: Functional and biochemical analysis of the cloned Duffy antigen: Identity with the red blood cell chemokine receptor. Blood 84: 44-52, 1994.

23. Graham GJ, Locati M, Mantovani A, Rot A and Thelen M: The biochemistry and biology of the atypical chemokine receptors. Immunol Lett 145: 30-38, 2012.

24. Ulvmar MH, Hub E and Rot A: Atypical chemokine receptors. Exp Cell Res 317: 556-568, 2011.

25. Wang D, Dubois RN and Richmond A: The role of chemokines in intestinal inflammation and cancer. Curr Opin Pharmacol 9: 688-696, 2009

26. Addison CL, Belperio JA, Burdick MD and Strieter RM: Overexpression of the duffy antigen receptor for chemokines (DARC) by NSCLC tumor cells results in increased tumor necrosis. BMC Cancer 4: 28, 2004.

27. Lentsch AB: The Duffy antigen/receptor for chemokines (DARC) and prostate cancer. A role as clear as black and white? FASEB J 16: 1093-1095, 2002.

28. McClellan JL, Davis JM, Steiner JL, Enos RT, Jung SH, Carson JA, Pena MM, Carnevale KA, Berger FG and Murphy EA: Linking tumor-associated macrophages, inflammation, and intestinal tumorigenesis: Role of MCP-1. Am J Physiol Gastrointest Liver Physiol 303: G1087-G1095, 2012.

29. Doll D, Keller L, Maak M, Boulesteix AL, Siewert JR, Holzmann B and Janssen KP: Differential expression of the chemokines GRO-2, GRO-3, and interleukin-8 in colon cancer and their impact on metastatic disease and survival. Int J Colorectal Dis 25: 573-581, 2010.

30. Ning Y, Manegold PC, Hong YK, Zhang W, Pohl A, Lurje G, Winder T, Yang D, LaBonte MJ, Wilson PM, et al: Interleukin-8 is associated with proliferation, migration, angiogenesis and chemosensitivity in vitro and in vivo in colon cancer cell line models. Int J Cancer 128: 2038-2049, 2011.

31. Wang J, Ou ZL, Hou YF, Luo JM, Chen Y, Zhou J, Shen ZZ, Ding J and Shao ZM: Duffy antigen receptor for chemokines attenuates breast cancer growth and metastasis: An experiment with nude mice. Zhonghua Yi Xue Za Zhi 85: 2033-2037, 2005.

32. Zeng XH, Ou ZL, Yu KD, Feng LY, Yin WJ, Li J, Shen ZZ and Shao ZM: Coexpression of atypical chemokine binders (ACBs) in breast cancer predicts better outcomes. Breast Cancer Res Treat 125: 715-727, 2011.

33. Sun G, Wang Y, Zhu Y, Huang C and Ji Q: Duffy antigen receptor for chemokines in laryngeal squamous cell carcinoma as a negative regulator. Acta Otolaryngol 131: 197-203, 2011.

34. Bandyopadhyay S, Zhan R, Chaudhuri A, Watabe M, Pai SK, Hirota S, Hosobe S, Tsukada T, Miura K, Takano Y, et al: Interaction of KAI1 on tumor cells with DARC on vascular endothelium leads to metastasis suppression. Nat Med 12: 933-938, 2006. 